\title{
SURFACE FUNCTIONALIZATION OF THE BIOLOGICAL GOLD NANOPARTICLES FOR MICRO-RNA TARGETING
}

\author{
${ }^{1}$ Parastoo POURALI, ${ }^{1}$ Oldrich BENADA, ${ }^{1}$ Veronika BENSON ${ }^{*}$ \\ ${ }^{1}$ Institute of Microbiology, Czech Academy of Sciences, Czech Republic, EU, \\ parastoo.pourali@biomed.cas.cz, benada@biomed.cas.cz, $\underline{\text { benson@biomed.cas.cz }}$
}

https://doi.org/10.37904/nanocon.2021.4358

\begin{abstract}
Among non-viral gene carriers with low toxicity and high transfection efficiency, the use of gold nanoparticles (AuNPs) is of particular interest due to their biocompatibility and special properties. This is the first time we attempted to functionalize the surface of the biological AuNPs in order to conjugate them with antimiR-135b through electrostatic interactions and knockdown the microRNA-135b gene expression inside the cells. A fungal strain, Fusarium oxysporum, was cultured in Sabouraud Dextrose Broth (SDB), centrifuged, and the mycelium-free supernatant was challenged with $1 \mathrm{mmol}$ final concentration of $\mathrm{HAuCl}_{4} \cdot 3 \mathrm{H}_{2} \mathrm{O}$ and incubated for $24 \mathrm{~h}$ at $37^{\circ} \mathrm{C}$ in a shake flask. AuNPs were characterized by visible spectrophotometry, Transmission Electron Microscopy (TEM), Scanning Electron Microscopy (SEM), Energy-Dispersive X-ray spectroscopy (EDS), and a zetasizer. The washed and sterilized AuNPs were used for cytotoxicity and conjugation assays. First transferrin (Tf) and then polyethylenimine (PEI) were used to functionalize and change the surface charge of the AuNPs and then antimiR-135b was conjugated to the AuNPs trough electrostatic interactions. Their association was confirmed by visible spectrophotometry and electrophoresis. Confocal microscopy was used to investigate the internalization of the AuNPs-antimiR-135b complex. The results proved the formation of AuNPs with a maximum absorption peak at $528 \mathrm{~nm}$, round and oval shapes $(15-20 \mathrm{~nm})$, and average zeta potential of $-21.02 \mathrm{mV}$. The AuNPs-antimiR-135b showed delayed electrophoresis unlike antimiR-135b or AuNPs alone. Functionalized AuNPs did not cause any toxicity in cell culture and confocal microscopy showed successful transfection of AuNPs-antimiR-135b into the vast majority of $4 \mathrm{~T} 1$ cells. We concluded that the biological AuNPs were non-toxic and they could carry antimiR-135b to enable gene silencing.
\end{abstract}

Keywords: Gold nanoparticles (AuNPs), Biological method, Conjugation, AntimiR-135b

\section{INTRODUCTION}

Various viral and non-viral carriers have been introduced for gene delivery applications. The use of viral vectors has some disadvantages, such as their immunogenicity, toxicity, and problems in scaling up for use in the medical field [1]. In addition, viral vectors cannot infect non-dividing cells. The second group, non-viral carriers such as nanoparticles (liposomes, magnetic nanoparticles and carbon nanotubes) [2-5] have been studied more because of their safety, ease of modification, long circulation time, and good utility [6,7]. Their disadvantages are high cytotoxicity and that they are hindered by several mechanisms leading to their low transfection efficiency. Therefore, researchers have attempted to develop cationic molecules that can form complexes with the negatively charged nucleic acids [8]. Among non-viral carriers with low toxicity and high transfection efficiency, the use of gold nanoparticles (AuNPs) is of particular interest due to their biocompatibility and special properties. It has been shown that the surface of AuNPs can be easily modified to achieve better gene delivery. Although, for example, the cationic polymer polyethylenimine (PEI) is usually considered the gold standard for gene delivery [9], it was shown that the use of PEI coated with AuNPs showed a six fold higher transfection property in the monkey kidney cell line (COS -7) than PEI-25 KDa as control [10]. There are some other studies that showed similar results [11-13]. AuNPs modified with cationic quaternary ammonium groups or 2-aminoethanethiol were able to bind to plasmid DNA by electrostatic binding [5, 14, 15]. 
AuNPs modified with cetyltrimethylammonium bromide (CTAB) to bind to siRNA have also been reported. AuNPs have been modified for targeted gene transfer so that they can bind to the specific target on the surface of the cells and carry the genes into the target cells. This type of modification increases the efficiency of gene delivery compared to normal gene delivery [16]. For example, AuNPs conjugated to transferrin (Tf, for cell targeting) and a DNA plasmid have been shown to have a high transfection rate in vitro and in vivo [17]. In addition to the numerous advantages of AuNPs used in this field, the main disadvantage is their toxicity. This type of nanoparticles is mainly produced by chemical and then by physical methods. In the production of AuNPs for gene delivery, the other method called biological technique has not been explored yet. In this approach, the nanoparticles are produced by different types of microorganisms as well as plants. The biologically produced nanoparticles can be formed inside or outside the cells, depending on the method used. It has been shown that some active groups of enzymes as well as polymers or polysaccharides are responsible for the reduction of toxic ions, introduced into the microbial cells, while turning them into less toxic nanoparticles. The biological technique for the production of nanoparticles is safe, fast, simple, environmentally friendly and inexpensive. The main advantage of biologically produced nanoparticles is their non-toxicity [18]. In this study, we used a fungal strain, Fusarium oxysporum, for the extracellular production of AuNPs. The zeta potential of the produced AuNPs was analyzed and to promote the interaction of AuNPs with antimiR135b (synthetic ssRNA complementary to endogenous target microRNA-135b), PEI was used to change the surface charge of AuNPs. We conjugated Tf to the AuNPs surface to accelerate targeted gene delivery. This was the first time that attempts were made to alter the surface charge of biological AuNPs to conjugate them with antimiR-135b through electrostatic interactions. The accomplishment of this technique was demonstrated by the detection of antimiR-135b in the cells, indicating successful gene transfer. The antimiR-135b alone could not transfect the cells. Following the AuNPs-antimiR-135b internalization, target microRNA-135b is found and silenced by antimiR-135b.

\section{MATERIALS AND METHODS}

\subsection{AuNPs Production and Characterization}

Fusarium oxysporum (CCF 3732, Czech Republic) was cultured in Sabouraud Dextrose Broth (SDB, Sigma Aldrich, USA) at $30^{\circ} \mathrm{C}$ in a shake flask for 1 week. The culture was centrifuged ( $2000 \mathrm{~g}$ for $5 \mathrm{~min}$ ) and the mycelia-free supernatant was used to prepare AuNPs [19]. In order to AuNPs production, the pH of the mycelium-free supernatant was adjusted to $10.100 \mathrm{ml}$ of the mycelium-free supernatant was mixed with 1 mmol final concertation of $\mathrm{HAuCl}_{4} 3 \mathrm{H}_{2} \mathrm{O}$ (Sigma Aldrich, USA) and incubated for $24 \mathrm{~h}$ at $37^{\circ} \mathrm{C}$ in a shake flask. The negative control flask (sterile SDB containing the same concentration of $\mathrm{HAuCl}_{4} .3 \mathrm{H}_{2} \mathrm{O}$ was also incubated [19].

The color change of the reaction mixture is the first sign of AuNPs production due to the localized surface plasmon resonance (LSPR) of AuNPs [20]. The produced AuNPs were washed three times with RNase-free $\mathrm{ddH}_{2} \mathrm{O}$ ( $\mathrm{pH}$ 7.4, Qiagen, Hilden, Germany). The tube was centrifuged at $20000 \mathrm{rpm}$ for $30 \mathrm{~min}$ and the pellet was dissolved in $1000 \mu \mathrm{l}$ Rase-free $\mathrm{ddH}_{2} \mathrm{O}$ [20].

\subsubsection{Visible Spectrophotometry}

The maximum absorption peak of AuNPs was determined using the Nanodrop spectrophotometer (Thermo Fisher Scientific, Massachusetts, USA). The sample was tested between 400-750 nm (blank sample was $\mathrm{ddH}_{2} \mathrm{O}$ ). The production would be proved if the maximum absorption peak was seen between $500-550 \mathrm{~nm}$ due to the LSPR of AuNPs [20].

\subsubsection{Transmission Electron Microscopy (TEM)}

Five microliters of AuNPs suspension were applied onto a glow discharge activated ( $30 \mathrm{~s}, 1 \mathrm{kV}, 10 \mathrm{~mA}$, [21]) carbon/formvar coated 300 mesh copper grids and let adsorb for $30 \mathrm{~s}$. Next followed the blotting the excess of the sample with filter paper and air-drying the grids at room temperature. Dried grids were examined at 80 
kV in the Philips CM100 TEM (Philips EO, Eindhoven, The Netherlands) equipped with a Veleta slow-scan CCD camera (EMSIS, Muenster, Germany).

\subsubsection{Scanning Electron Microscopy (SEM) and Energy-Dispersive X-ray Spectroscopy (EDS)}

Ten microliters of AuNPs suspension were dried out overnight at $56{ }^{\circ} \mathrm{C}$ on a glow discharge activated silicon wafer. SEM and EDS analyses were performed using FEI Nova Nano SEM 450 scanning electron microscope ( $\mathrm{FEl}$, Brno, Czech Republic) equipped with an Ametek ${ }^{\circledR}$ EDAX Octane, SDD detector, and TEAM ${ }^{\mathrm{TM}}$ EDS Analysis Systems (AMETEK B. V.; Tilburg, The Netherlands) essentially as described in [22].

\subsubsection{Zetasizer Analysis}

The average size and zeta potential of AuNPs were determined using Zetasizer Nano ZS90 (Malvern Panalytical, Malvern, UK) and DLS (Dynamic Light Scattering, by noninvasive backscattering technique) instruments in the presence of $\mathrm{ddH}_{2} \mathrm{O}$ as dispersant and $25^{\circ} \mathrm{C}$ [23].

\subsection{Cytotoxicity Test}

Before the cytotoxicity test the nanoparticles were sterilized using the Tyndallization technique according to Pourali et al [19]. MTT assay was used to determine the toxicity of AuNPs before any other experiments. Two different cell cultures, NIH/3T3 (ATCC CRL-1658) and 4T1 (ATCC CRL-2539), were used as control (normal mouse fibroblasts) and test (BALB/c mouse breast cancer cell line), respectively. NIH/3T3 and 4T1 cells were cultured in Dulbecco's Modified Eagle Medium (DMEM, Sigma Aldrich, USA) and Roswell Park Memorial Institute Medium 1640 (RPMI-1640, Sigma Aldrich, USA), respectively. Both in the presence of $10 \%$ Fetal Bovine Serum (FBS, Gibco, Massachusetts, USA) and $44 \mu \mathrm{g} / \mathrm{ml}$ gentamicin (Sandoz, Novartis Company, Prague, Czech Republic). In each well of 96 wells bottom tissue culture plate (JETBiofil, Guangzhou, China), $2 \times 10^{5}$ cells were seeded and after reaching $80 \%$ confluence monolayer cells, cells were washed with phosphate buffered saline (PBS) and all wells examined were filled with $200 \mu \mathrm{l}$ of the fresh medium. AuNPs were $1 / 2$ diluted by titration in the $B 2$ line in fresh medium and then the diluted samples were used. In well $A 1$, $50 \mu \mathrm{L}$ of AuNPs were added and the same amount of AuNPs, but diluted, was added in A2 to A11. Well A12 was the positive control without addition of AuNPs. The plate was incubated overnight in a humid atmosphere, $5 \% \mathrm{CO}_{2}$ incubator at $37^{\circ} \mathrm{C}$ and then $20 \mu \mathrm{L}$ of $5 \mathrm{mg} / \mathrm{mL}$ (3-4,5-dimethylthiozol-2-yl) 2,5-diphenyltetrazolium bromide (MTT, EMD Millipore, CA, USA) in PBS ( $\mathrm{pH} 7.4$ ) was added to all wells. After an incubation period of 4 hours, the mixture was removed and $100 \mu \mathrm{L}$ of dimethyl sulfoxide (DMSO, Sigma Aldrich, USA) was added. The absorbance of the wells was measured using a Tecan spectrophotometer (Thermo Fisher Scientific, Massachusetts, USA) at a wavelength of $570 \mathrm{~nm}$. The percentage of half-maximal inhibitory concentration $\left(\mathrm{IC}_{50}\right)$ was calculated for each cell line using the following formula: $\mathrm{IC}_{50}=\mathrm{OD}$ of maximum inhibition - 50\% (OD of maximum inhibition - OD of minimum inhibition) [20].

\subsection{Functionalization and Conjugation}

To functionalize AuNPs with Tf and PEI, $200 \mu \mathrm{l}$ AuNPs (at a non-toxic concentration according to MTT assay result) were incubated with $200 \mu \mathrm{lff}(0.9 \mu \mathrm{g} / \mathrm{ml}$; labeled with Texas Red, Life Technologies, Prague, Czech Republic) for $1 \mathrm{~h}$ with shaking at room temperature (RT). $400 \mu \mathrm{l}$ of low weight PEI 800 (Sigma-Aldrich, Prague, Czech Republic) was added to the sample and the mixture was incubated overnight at RT. Unbound molecules ( $\mathrm{Tf}$ and $\mathrm{PEI}$ ) were washed with $\mathrm{RNase}-$ free $\mathrm{ddH}_{2} \mathrm{O}$ and removed by centrifugation twice at $9000 \mathrm{~g}$ for $60 \mathrm{~min}$. The pellet was dissolved in $400 \mu \mathrm{l}$ of RNase-free $\mathrm{ddH}_{2} \mathrm{O}$. To conjugate the antimiR-135b to the surfacefunctionalized AuNPs, $36 \mu \mathrm{l}$ of antimiR-135b: 5'UptCptAptCAUAGGAAUGAAAAGCCptAptUptA 3' (labeled with Alexa Fluor 488 at $5^{\prime}$ ) was added and incubated at RT for $1 \mathrm{~h}$ under shaking conditions. Unbound antimiR$135 \mathrm{~b}$ molecules were removed by centrifugation at $9000 \mathrm{~g}$ for $60 \mathrm{~min}$. The pellet was suspended in $400 \mu \mathrm{l}$ RNase-free $\mathrm{ddH}_{2} \mathrm{O}$ and used for in vitro assay [24]. To detect the conjugation of antimiR-135b to AuNPs, different methods were used. Firstly, the maximum absorption peak of antimiR-135b-AuNPs was checked by 
spectrophotometry. The change in the curve compared to AuNPs as control was evidence of successful functionalization and conjugation. The blank value used was $\mathrm{ddH}_{2} \mathrm{O}$ [20]. Second, agarose gel electrophoresis was performed for antimiR-135b-AuNPs, antimiR-135b (as control) and MassRuler low range DNA Ladder (Thermo Fisher Scientific, Massachusetts, USA). Tris-acetate-EDTA buffer (TAE) was used as buffer and the gel of $2 \% \mathrm{w} / \mathrm{v}$ agarose (Sigma Aldrich, USA) was prepared. $5 \mu \mathrm{L}$ of each sample was mixed with $1 \mu \mathrm{L}$ of $6 \mathrm{X}$ Mass Ruler DNA loading dye (Thermo Fisher Scientific, Massachusetts, USA) and added to the wells of the gel. The gel was run at a voltage of $100 \mathrm{~V}$ for $45 \mathrm{~min}$ and visualized under gel documentation. Successful conjugation results in a delay in the migration of antimiR-135b-AuNPs compared to antimiR-135b as a control [25-27]. Third, the zeta potential and size distribution of antimiR-135b-AuNPs were determined using the same technique as described previously.

\subsection{Confocal Microscopy}

4T1 cells were cultured in 35-mm dishes with 20-mm glass bottoms (Cellvis, Ontario, Canada) for $12 \mathrm{~h}$ under culture conditions described previously. After the cells were $80 \%$ confluent, the medium from each plate was discarded and $1500 \mu \mathrm{L}$ of fresh medium was added, and $60 \mu \mathrm{L}$ of antimiR-135b-AuNPs sample was added for the test plate. After 24 hours of incubation, cells were washed three times with PBS and immediately analyzed live under an Olympus FluoView FV1000 confocal microscope (objectives 20X/ NA 0.75 and 40X/ NA 0.95) without fixation or staining. Excitation/emission parameters: Alexa Fluor 488 473/520 nm; TexasRed 595/615 $\mathrm{nm}$. Results were analyzed using Olympus FluoView 2.0 software [24].

\section{RESULTS AND DISCUSSION}

\subsection{AuNPs Production and Characterization}

After 24 hours of incubation, the mixture turned from yellow to red due to the LSPR of AuNPs. Figure 1 B shows the color change compared to the negative control (A). As mentioned earlier, the $\mathrm{pH}$ of the myceliumfree supernatant was adjusted to 10 because an alkaline environment is reportedly preferred, as the repulsion between $\mathrm{Au}^{+}$and $\mathrm{H}^{+}$ions at acidic $\mathrm{pH}$ values reduces the probability of reduction and nucleation of $\mathrm{Au}^{+}$ions to AuNPs [28].

\subsubsection{Visible Spectrophotometry}

The maximum absorption peak of AuNPs was reached at $528 \mathrm{~nm}$ (the blank sample was $\mathrm{ddH}_{2} \mathrm{O}$ ), which was the evidence of the production of AuNPs in the sample. Figure $1 \mathbf{C}$ shows the results obtained.
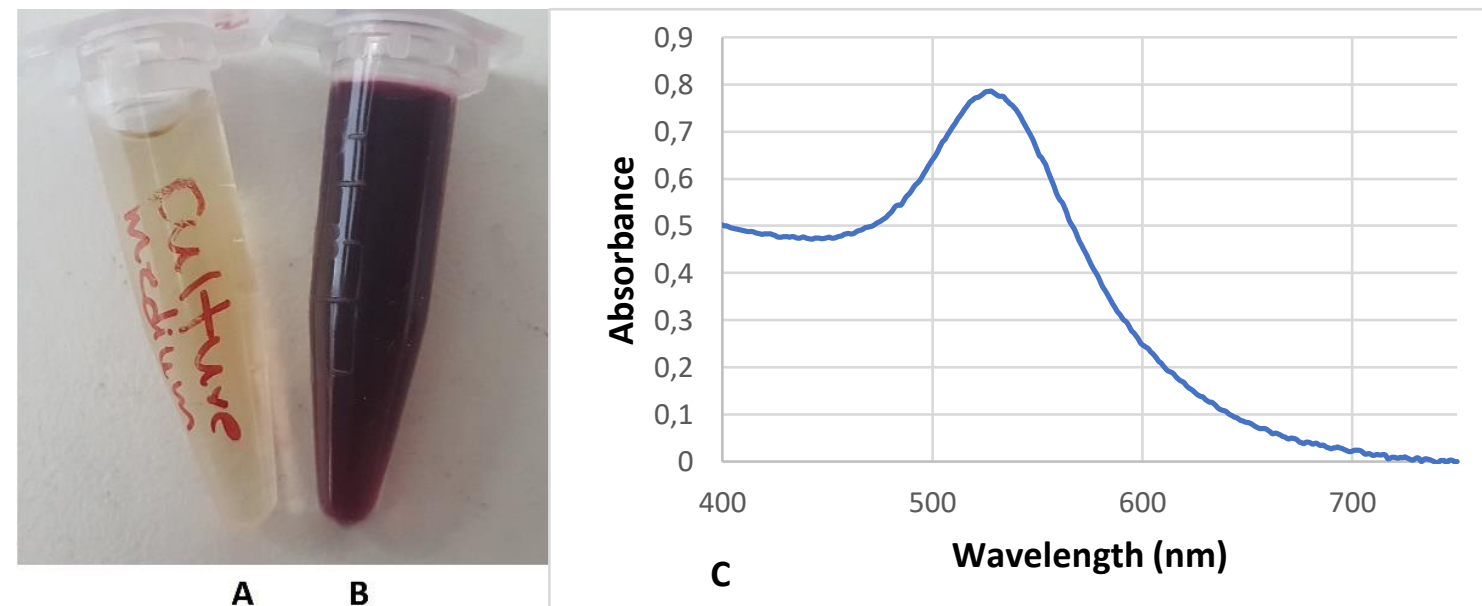

Figure 1 Production and characterization of biologically produced AuNPs. A) Control B) The color-changed mycelia-free supernatant. C) The obtained AuNPs spectrum with maximum absorption peak at $528 \mathrm{~nm}$ 


\subsubsection{Electron Microscopy of AuNPs}

Figure 2 shows the representative frame of TEM AuNPs imaging that proved the spherical to oval shape and almost the same size $(15-20 \mathrm{~nm})$ of analyzed particles. TEM analysis also proved the purity of the prepared AuNPs sample.

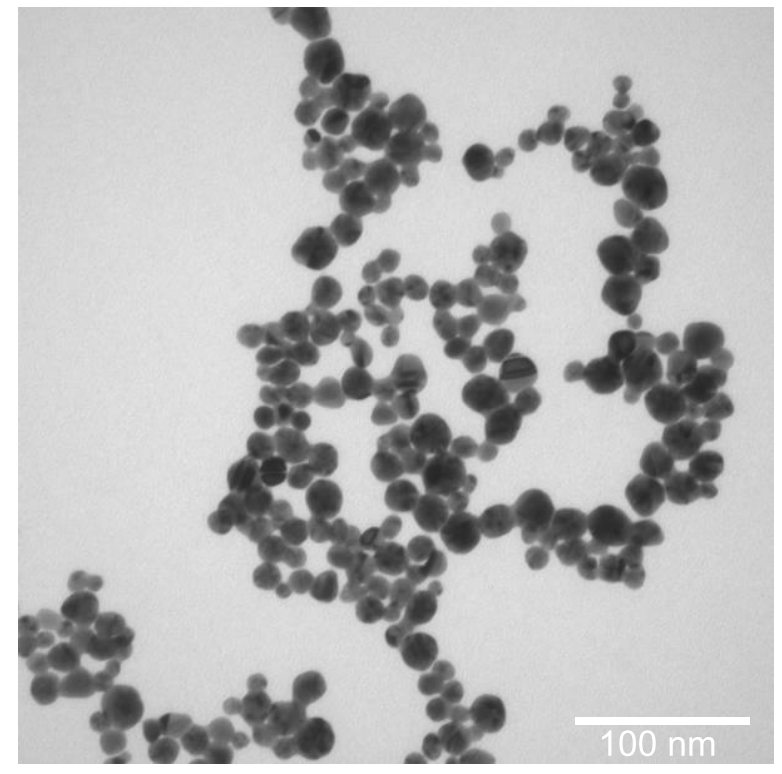

Figure 2 TEM image of AuNPs on glow discharge activated carbon/formvar support film. The particles possess a spherical shape with an approximate size of $20 \mathrm{~nm}$. Scale bar $=100 \mathrm{~nm}$.

Figure 3 A shows a backscattered electron SEM image of well-spread AuNPs with not many aggregated clusters on the wafer surface. The glow-discharge activation of the silicon wafer surface worked quite well and minimized the artificial clustering of AuNPs.

The EDS spectrum (Figure 3 B) confirmed the Au content in the imaged nanoparticles showing characteristic $\mathrm{Au} \mathrm{M} \alpha$ and $\mathrm{M} \beta$ peaks at the energies of $2.123 \mathrm{keV}$ and $2.203 \mathrm{keV}$. The insert in Figure $\mathbf{3} \mathbf{B}$, an SEM secondary electron image, shows the approximate location of the electron beam during the EDS spectrum acquisition, marked with a white letter $X$.
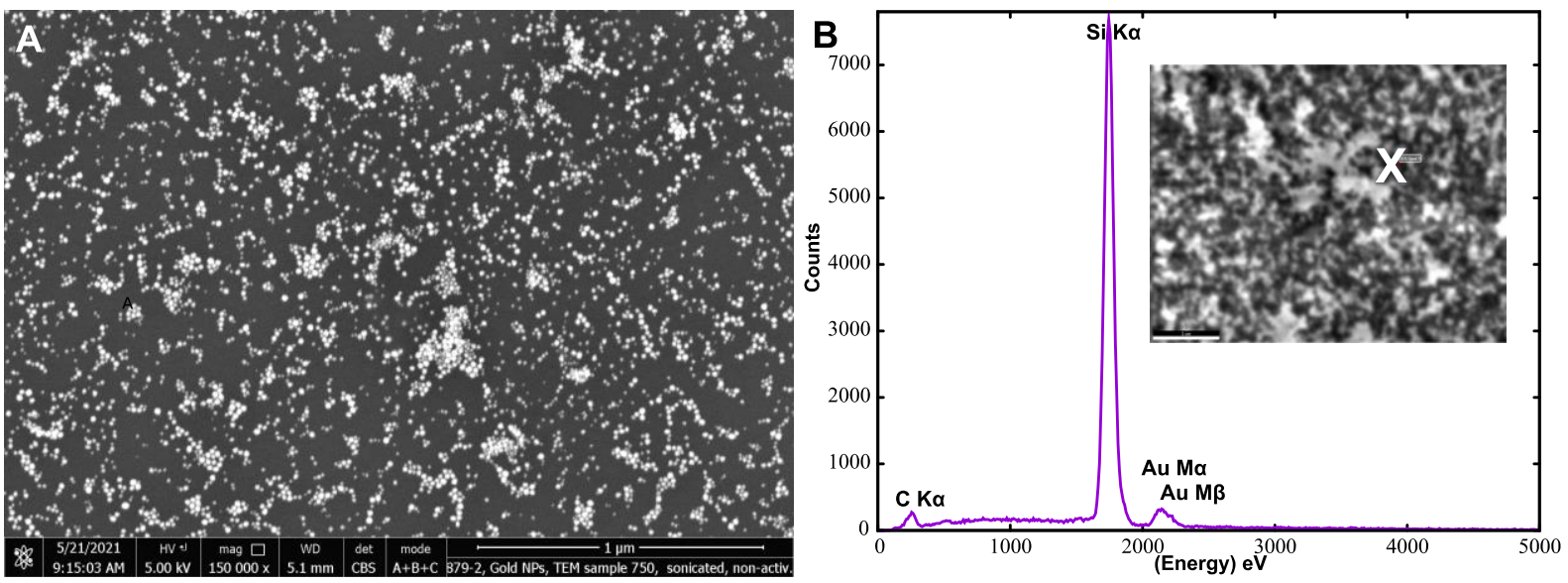

Figure 3 SEM and EDS analyses of Au NPS. A) SEM back-scattered electron image and B) EDS spectrum and spot location in secondary electron image. Spectrum acquisition conditions - Spot size: 5 ; Accelerating voltage: 6 kV; Magnification: 65000 ×; Take-off angle: 35; Live Time: 30 s; Amp Time: 7.68 us; Resolution: $128.1 \mathrm{eV}$. Scale bar $=1 \mu \mathrm{m}$. 
The other peaks in the spectrum represent the Si Ka signal at the energy of $1.740 \mathrm{keV}$, which originates from silicon wafer support, and the $\mathrm{C} \mathrm{Ka}$ signal at the energy of $0.277 \mathrm{keV}$. The carbon signal probably reflects the presence of organic capping on the AuNPs' surface. The AuNPs sample was prepared from mycelium-free supernatant and extensively washed before sample preparation for SEM. It minimized the contamination of AuNPS with culture medium or the remnants of fungal mycelium.

\subsubsection{Zetasizer analysis}

The results show that the hydrodynamic diameter ( $Z$ average) of AuNPs is $49.81 \pm 4 \mathrm{~nm}$ and the zeta potential is -21.02 . The obtained $Z$-average was higher than the results of TEM, $49.81 \pm 4 \mathrm{~nm}$ compared to $20 \mathrm{~nm}$. Thus, the Z-average result from the digital images of TEM is different from DLS because DLS measures the hydrodynamic diameter and TEM measures only the diameter of AuNPs [27].

\subsection{Cytotoxicity assay}

MTT assay was used to determine the toxicity of AuNPs before any other experiments. The results showed that the AuNPs did not induce toxic effects in NIH/3T3 and 4T1 cell cultures. The result is consistent with previous studies that reported that this type of nanoparticles produced by microorganisms has dose-dependent and low toxic effects [18].

\subsection{Functionalization and Conjugation}

During the conjugation process, no significant color change of the samples was observed after the addition of the single molecules to the AuNPs, indicating that the nanoparticles were stable during the functionalization process. After functionalization of AuNPs with Tf and PEI and conjugation with antimiR-135b, the maximum absorption peak was determined. Figure 4 shows the differences between the maximum absorption peaks of AuNPs $(528 \mathrm{~nm}$ ) versus antimiR-135b-AuNPs $(533 \mathrm{~nm})$. As can be seen (Figure 4 A), the peak of the control was sharper in contrast to the antimiR-135b-AuNPs. Moreover, the absorption maximum of the AuNPs after conjugation with these molecules was at a higher wavelength, but in the range of 500-550 nm. This means that the AuNPs were stable during the conjugation process and remained in their nanoform. The broader peak and the change in peak position (Figure $4 \mathbf{A}$ ) indicate that the AuNPs conjugated with these molecules.
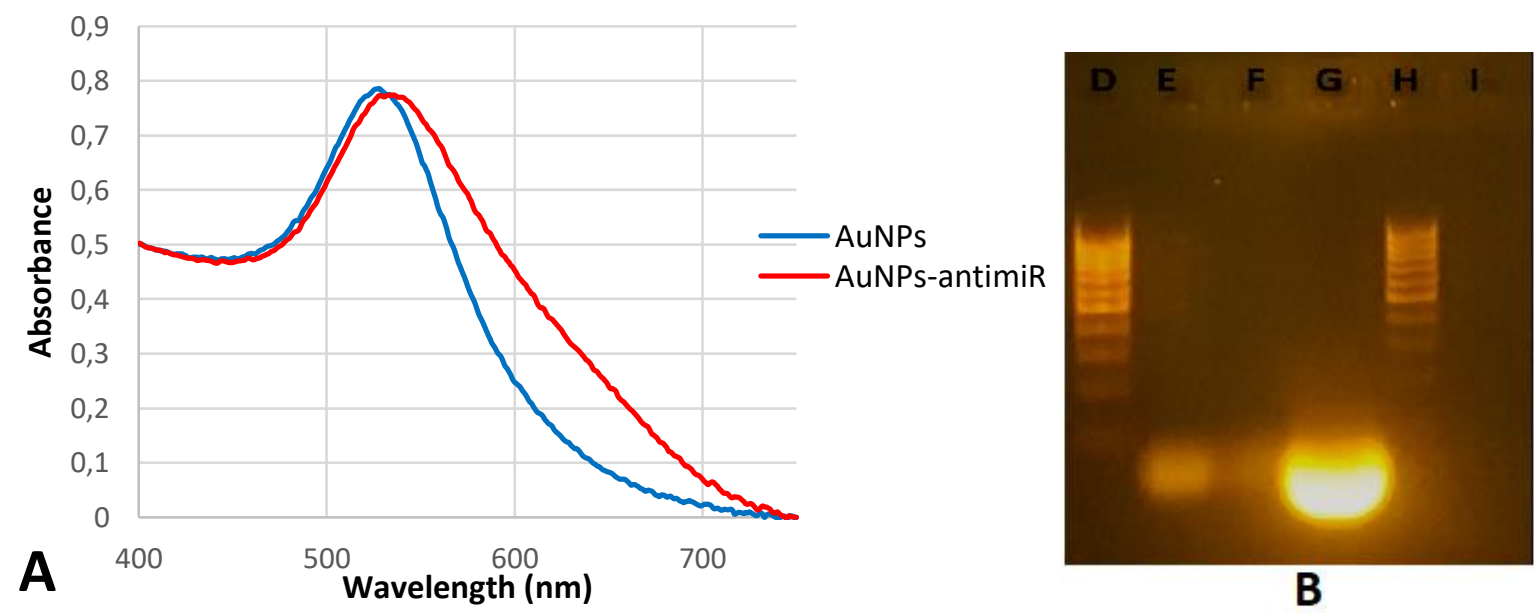

B

Figure 4 Detection of conjugation by visible spectrophotometry and agarose gel electrophoresis. Blue peak is AuNPs as control and red peak belongs to antimiR-135b AuNPs. (B) Agarose gel electrophoresis results. Lanes $D$ and $H$ are DNA ladder, lanes $E$ and $F$ are antimiR-135b-AuNPs with two different loads, lane $G$ is antimiR-135b and lane I is AuNPs.

Agarose gel electrophoresis was performed for antimiR-135b-AuNPs and antimiR-135b. The results show the delayed migration of antimiR-135b-AuNPs compared to antimiR-135b as control. Figure $4 \mathbf{B}$ shows the results 
obtained. A short delay can be seen in lanes $E$ and $F(10$ and $5 \mu$ l were loaded respectively) in contrast to the control (lane G). This means that after conjugation of antimiR-135b with AuNPs, since this complex is heavier than antimiR-135b as control, it is seen in the higher position in the gel. The zetasizer results showed that the $Z$ average of antimiR-135b-AuNPs was $53 \pm 7 \mathrm{~nm}$ with a zeta potential of $+10.53 \mathrm{mV}$. The obtained $Z$ average was higher than that of AuNPs, indicating the changes on the surface of AuNPs after conjugation with the corresponding molecules.

All in all, the obtained data confirmed that the surface charge of the biologically prepared aunps changed from negative to positive after pei treatment and the nanoparticles were ready for conjugation with antimir-135b. Thus, the functionalization of the aunps was successful. We have previously reported that direct conjugation of the biological aunps with various drugs such as antibiotics is possible $[19,20]$. In the current study, we have shown that the surface charge of the nanoparticles can be easily changed so that they are ready to bind to the nucleic acids through electrostatic interactions and without additional linkers.

\subsection{Confocal Microscopy}

Confocal microscopy results showed successful transfection of antimiR-135b-AuNPs into the vast majority of $4 \mathrm{~T} 1$ cells (Figure 5).

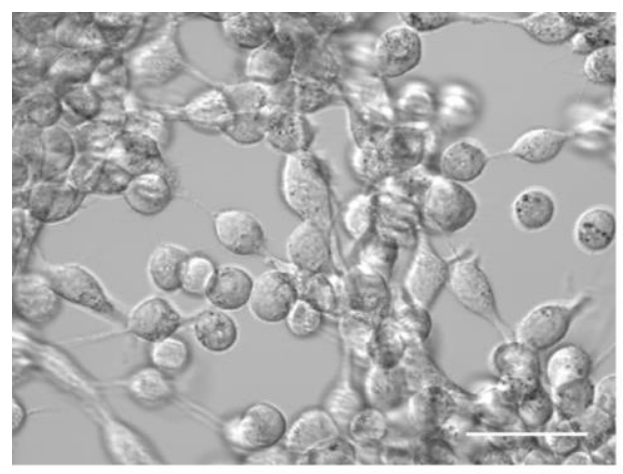

A

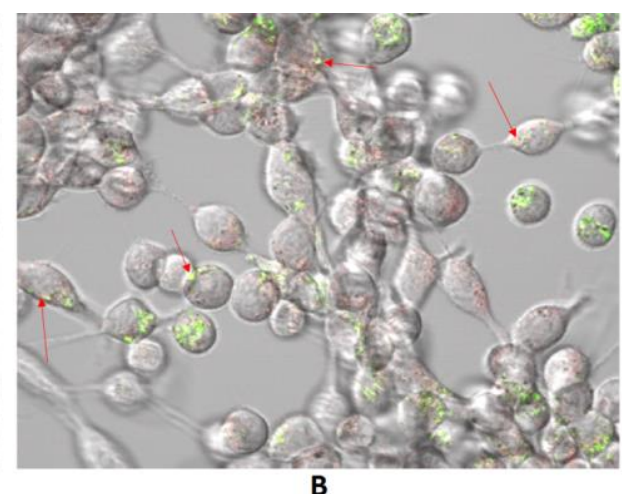

B

Figure 5 Results of confocal microscopy of live 4T1 cells after transfection with antimiR-135b-AuNPs. A) the cells as background and $B$ ) the internalized complexes in the cells. The red signal is antimiR-135b and the green signal is Tf. The arrows show some of the complexes. Scale bar $=100 \mu \mathrm{m}$.

\section{CONCLUSION}

This was the first study of possible functionalization of AuNPs produced by the biological method, which was completely unknown before. The biological AuNPs were non-toxic and can be functionalized to bind antimiR$135 \mathrm{~b}$ to enable gene silencing. Our results showed that antimiR-135b could attach to the positively charged nanoparticles after the AuNPs were functionalized with $\mathrm{Tf}$ and PEI. Confocal microscopy results showed that the nanoparticles were taken up into the target cells. Thus, the functionalization of the biological AuNPs made them a good candidate for targeted gene silencing in the cells. Further studies are needed to investigate the other capabilities of the biological AuNPs.

\section{ACKNOWLEDGEMENTS}

This work was supported by ESF "International Mobility of Researchers - MSCA-IF IV (Institute of Microbiology of CAS, v. v. i.) No. CZ.02.2.69/0.0/0.0/20_079/0017812. We thank CMS-Biocev ("Biophysical techniques, crystallization, diffraction, structural mass spectrometry") of CIISB, center Instruct-CZ Centre, supported by MEYS CR (LM2018127). 


\section{REFERENCES}

[1] VERMA, I.M., et al. Gene therapy: promises, problems and prospects. Genes and Resistance to Disease. 2000, pp. 147-157.

[2] BOYER, C., et al. Anti-fouling magnetic nanoparticles for siRNA delivery. Journal of Materials Chemistry. 2010, vol. 20, no. 2, pp. 255-265.

[3] GAO, L., et al. Carbon nanotube delivery of the GFP gene into mammalian cells. ChemBioChem. 2006, vol. 7, no. 2, pp. 239-242.

[4] SUZUKI, R., et al. Effective gene delivery with novel liposomal bubbles and ultrasonic destruction technology. International journal of pharmaceutics. 2008, vol. 354, no. 1-2, pp. 49-55.

[5] MCINTOSH, C.M., et al. Inhibition of DNA transcription using cationic mixed monolayer protected gold clusters. Journal of the American Chemical Society. 2001, vol. 123, no. 31, pp. 7626-7629.

[6] BRANCO, M.C. SCHNEIDER, J.P. Self-assembling materials for therapeutic delivery. Acta biomaterialia. 2009, vol. 5, no. 3, pp. 817-831.

[7] LIU, F. HUANG, L. Development of non-viral vectors for systemic gene delivery. Journal of controlled release. 2002, vol. 78, no. 1-3, pp. 259-266.

[8] MINTZER, M.A. SIMANEK, E.E. Nonviral vectors for gene delivery. Chemical reviews. 2009, vol. 109, no. 2, pp. 259-302.

[9] BOUSSIF, O., et al. A versatile vector for gene and oligonucleotide transfer into cells in culture and in vivo: polyethylenimine. Proceedings of the National Academy of Sciences. 1995, vol. 92, no. 16, pp. 7297-7301.

[10] THOMAS, M. KLIBANOV,A.M. Conjugation to gold nanoparticles enhances polyethylenimine's transfer of plasmid DNA into mammalian cells. Proceedings of the National Academy of Sciences. 2003, vol. 100, no. 16, pp. 91389143.

[11] NOH, S.M., et al. Enhanced cellular delivery and transfection efficiency of plasmid DNA using positively charged biocompatible colloidal gold nanoparticles. Biochimica et Biophysica Acta (BBA)-General Subjects. 2007, vol. 1770, no. 5, pp. 747-752.

[12] HU, C., et al. Low molecular weight polyethylenimine conjugated gold nanoparticles as efficient gene vectors. Bioconjugate chemistry. 2010, vol. 21, no. 5, pp. 836-843.

[13] RAHME, K., et al. Bioconjugated gold nanoparticles enhance siRNA delivery in prostate cancer cells. RNA interference and cancer therapy. 2019, pp. 291-301.

[14] HAN, G., et al. Stability of gold nanoparticle-bound DNA toward biological, physical, and chemical agents. Chemical biology \& drug design. 2006, vol. 67, no. 1, pp. 78-82.

[15] NIIDOME, T., et al. Preparation of primary amine-modified gold nanoparticles and their transfection ability into cultivated cells. Chemical communications. 2004, vol. 17, pp. 1978-1979.

[16] CHEN, P.C., et al. Gold nanoparticles: from nanomedicine to nanosensing. Nanotechnology, science and applications. 2008, vol. 1, pp. 45.

[17] SALEM, A.K., et al. Multifunctional nanorods for gene delivery. Nature materials. 2003, vol.. 2, no. 10, pp. 668671.

[18] POURALI, P., et al. Biosynthesis of gold nanoparticles by two bacterial and fungal strains, Bacillus cereus and Fusarium oxysporum, and assessment and comparison of their nanotoxicity in vitro by direct and indirect assays. Electronic Journal of Biotechnology. 2017, vol. 29, pp. 86-93.

[19] POURALI, P., et al. Bio-synthesis of gold nanoparticles by Fusarium oxysporum and assessment of their conjugation possibility with two types of $\beta$-lactam antibiotics without any additional linkers. Microbiology. 2018, vol. 87, no. 2, pp. 229-237.

[20] YAHYAEI, B., POURALI, P. One step conjugation of some chemotherapeutic drugs to the biologically produced gold nanoparticles and assessment of their anticancer effects. Scientific reports. 2019, vol. 9, no. 1, pp. 1-15.

[21] BENADA, O. POKORNY, V. Modification of the Polaron sputter-coater unit for glow-discharge activation of carbon support films. Journal of electron microscopy technique. 1990, vol. 16, no. 3, pp. 235-239.

[22] POURALI, P., et al. Response of Biological Gold Nanoparticles to Different pH Values: Is It Possible to Prepare Both Negatively and Positively Charged Nanoparticles? Applied Sciences. 2021, vol. 11, no. 23, pp. 11559-11579. 
[23] EATON, P., et al. A direct comparison of experimental methods to measure dimensions of synthetic nanoparticles. Ultramicroscopy. 2017, vol. no. 182, pp. 179-190.

[24] KRIVOHLAVA, R., et al. Knockdown of microRNA-135b in mammary carcinoma by targeted nanodiamonds: potentials and pitfalls of in vivo applications. Nanomaterials. 2019, vol. 9, no. 6, pp. 866.

[25] CUI, J., et al. Application of PEI-modified magnetic nanoparticles as gene transfer vector for the genetic modification of animals. Advances in Materials Science and Engineering. 2012, vol. 2012, pp. 1-6.

[26] GUO, C. YARGER, J.L. Characterizing gold nanoparticles by NMR spectroscopy. Magnetic Resonance in Chemistry. 2018, vol. 56, no. 11, pp. 1074-1082.

[27] SHAWKY, S.M., et al. Direct detection of unamplified hepatitis $C$ virus RNA using unmodified gold nanoparticles. Clinical biochemistry. 2010, 43, no.13-14, pp. 1163-1168.

[28] GURUNATHAN, S., et al. Biosynthesis, purification and characterization of silver nanoparticles using Escherichia coli. Colloids and Surfaces B: Biointerfaces. 2009, vol. 74, no. 1, pp. 328-335. 